\title{
Le débat sur le rationnement, une impossibilité politique (?)
}

\section{Jean Martin}

Ancien médécin cantonal vaudois, membre de la Commission nationale d'ethique

\section{* A ce propos, expérience vécue il y a quelques semaines lors d'une séance de comité d'une associa- tion nationale du domaine de la santé: un collègue a proposé que l'association crée un groupe de travail pour débattre du thème (menaçant) du rationne- ment. Un autre collègue, professionnellement actif dans une institution de soins et impliqué sociale- ment, a alors dit que, au contraire, nous devrions dire que nous sommes pour le rationnement, inévitable mais qu'il importe de concevoir dans un sens aussi équitable que possible! Bien que plusieurs membres voient le caractère raisonnable d'une telle proposition, inutile de dire que le comité n'envisage pas de signaler publiquement qu'il est favorable à un rationne- ment «socialement respectueux».}

En Suisse comme ailleurs, une demande croissante de soins, liée notamment à l'augmentation de l'éventail des offres envisageables de traitement, et les limites des moyens disponibles se conjuguent pour entraîner des difficultés chroniques pour le système de santé. Dans ce contexte, l'hypothèse d'un rationnement est souvent discutée: quant au principe, personne n'en veut mais nombreux sont ceux qui estiment que, explicite ou implicite, on ne pourra l'éviter - et que alors, autant s'efforcer de le réaliser dans des conditions et avec des critères qui soient les moins mauvais possibles. Daniel Callahan, fondateur du Hastings Center et figure majeure de la bioéthique outre-Atlantique, s'est penché à réitérées reprises sur ce sujet. Ceci dans le cadre d'un engagement fort, cela peut surprendre, pour plus d'équité dans l'accès aux soins, équité dont le «non-système» de santé US a bien besoin. Cela fait noter que, oui, dans nos sociétés un rationnement éventuel est une dimension de l'équité (comme a pu l'illustrer, dans un domaine différent, le programme national de rationnement durant la Seconde guerre mondiale). ${ }^{*}$

Tout récemment, Callahan a exprimé sa désillusion quant à la possibilité d'instaurer au niveau d'une collectivité un rationnement «raisonnable», acceptable [1]. Je cite:

«Finalement, le problème avec le rationnement est que nous avons une culture et un fonctionnement politique qui évite les dilemmes éthiques lourds, qui promeut l'expression d'indignations bruyantes plutôt qu'une véritable délibération démocratique. Nous sommes un cas grave de «California disease`: vouloir limiter les impôts tout en formulant des demandes illimitées de services. Nous voulons un progrès médical infini, une guerre inconditionnelle contre la mort et ceci sans rationnement. Cette situation n'est pas tenable longtemps mais, comme un microorganisme résistant aux traitements, elle connaît de constantes mutations et la société reste malade, d'une maladie économique chronique. Le refus de nos politiciens de parler ouvertement de la nécessité du rationnement [aussi désagréable cela soit-il] n'est pas moins pathologique.»

«La culture d'évitement de la réalité est en opposition frontale avec la nécessité de contrôler les coûts. Les forces politiques qui réclament le plus bruyamment la réduction des déficits publics sont cellesmêmes qui condamnent toute idée d'aborder le thème du rationnement.» «Alle Politiker entfliehen der Debatte, weil sie heikel ist», dit le conseiller natio- nal et médecin Ignazio Cassis à propos d'un cas récent dans notre pays (article de I. Städler cité sous [6]). Pour ma part, je me souviens n'avoir reçu qu'aimables fins de non-recevoir quand j'insistais auprès du Ministre cantonal de la santé - sous lequel je servais - pour que lui, le ministre, rappelle clairement que les moyens sont toujours rares et demandent des choix qui, d'une manière ou de l'autre, correspondent à un rationnement; par exemple parce que ceux qui vivent à proximité d'un Centre hospitalier universitaire ont plus de chances de recevoir sans délai le traitement optimal en cas de grande urgence. A mon sens, la réalité de la vie en communauté démocratique - où les décisions sont prises à la majorité mais s'imposent à tous - nous «condamne» à accepter ces faits.

\section{Accès au rein artificiel - Seattle, 1960}

Callahan rappelle un moment déterminant de l'irruption du rationnement sur la scène médicale occidentale, en 1960 à Seattle: le néphrologue Belding Scribner y avait développé et mis en œuvre le premier rein artificiel. Le nombre de machines étant très limité par rapport aux candidats potentiels, un «Admissions and Policy Committee» a été mis en place pour choisir, selon l'expression de plusieurs observateurs, qui vivra et qui mourra. Tentative en soi estimable de donner accès à une prestation aux malades pour qui c'est le plus judicieux/indiqué (position éthique de type utilitariste). Mais cela a créé une très vive polémique; des éthiciens éminents ont dit alors que, dans un tel cas, il vaudrait mieux tirer au sort (variante d'orientation déontologique). On peut relever que la question de critères du registre social (au sens large) dans les décisions d'attribution de ressources rares reste très présente dans les débats bioéthiques aujourd'hui - par exemple en ce qui concerne la transplantation d'organes.

\section{L'espoir déçu d'une délibération civique sur un rationnement transparent}

Seattle, c'était il y a 50 ans. Le problème reste actuel (pour la Suisse, voir [2, 3]). Pour Callahan, la doctrine générale qui émerge des réflexions faites jusqu'ici est la suivante: tôt ou tard le rationnement sera nécessaire; le rationnement direct au lit du malade n'est pas acceptable (trop sujet à biais et critères discutables); le rationnement devra être décidé au niveau des politiques de santé (policy), pour l'essentiel sans faire reposer le fardeau sur les médecins ou les pa- 


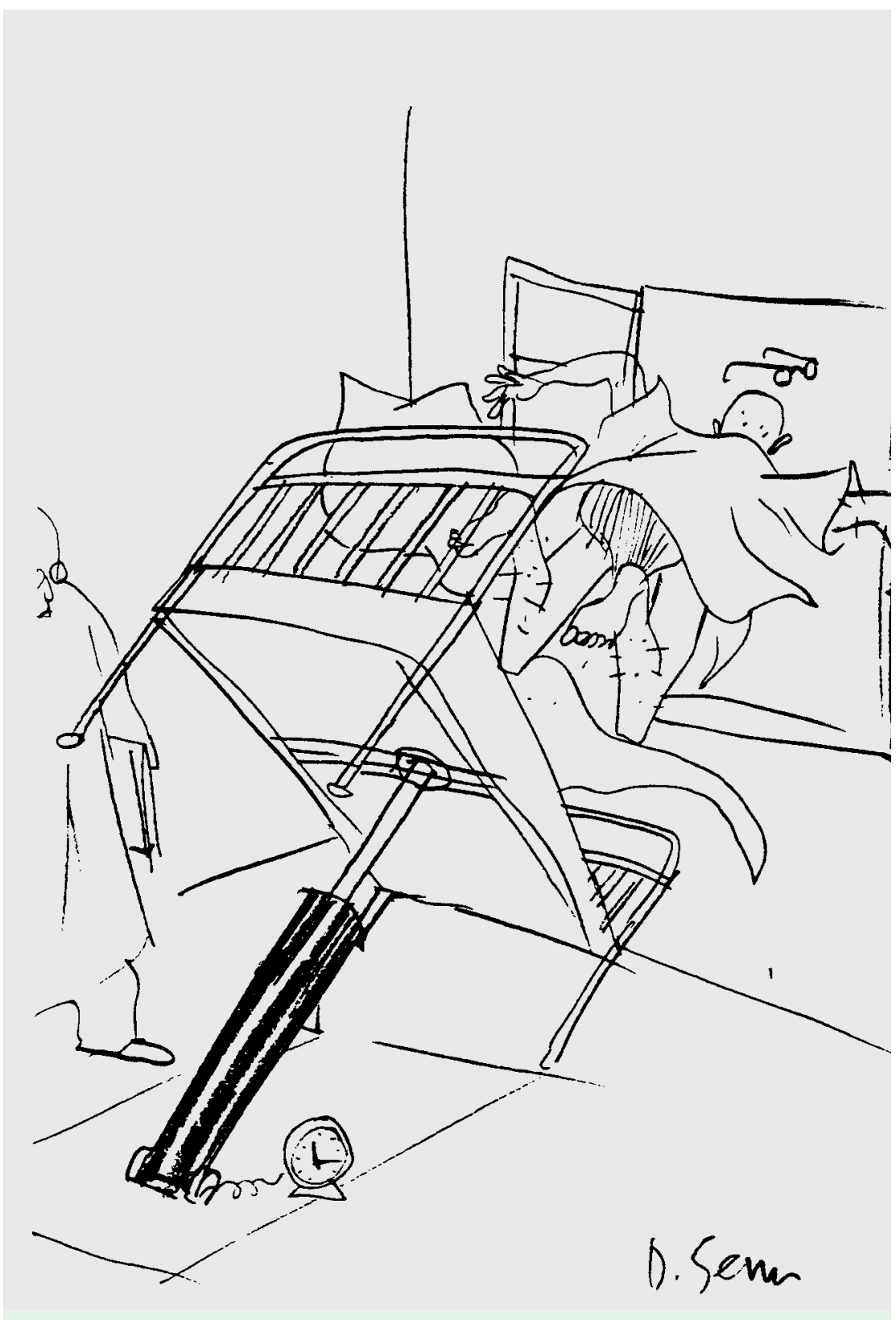

Une modalité future de rationnement? (Dessin de Daniel Senn)

tients; et il faudrait instituer des procédures de décision (des comités d'un type ou de l'autre) qui, après délibération, permettront des décisions explicites et transparentes».

Or, après des décennies de débat, il arrive à la conclusion que ce rationnement «transparent» ne pourra jamais être mis en œuvre dans les conditions socio-politiques américaines. «Trop de gens ne veulent aucun débat raisonnable sur le sujet. Comment organiser une discussion publique sensée si la moitié peut-être de nos concitoyens considèrent immoral le fait simplement d'en parler.» Pourtant, il y a des exemples de mise en œuvre d'un système de rationnement au niveau national, dans la clarté: en Nouvelle Zélande [4, 5];en Grande-Bretagne depuis des décennies de manière à la fois implicite et explicite; noter aussi le cas particulier de l'Oregon, qui a mis en place un système de liste où les prestations sont classées par ordre d'efficacité et économicité, et où l'endroit dans la liste où l'assurance-maladie subventionnée cesse de payer est déterminé par les ressources disponibles. Mais, pour les Etats-Unis (à part l'Oregon) comme à mon sens pour la Suisse ou la France, il y a refus - souvent tacite mais néanmoins catégorique - des personnalités et partis politiques de débattre de rationnement. Cette éventualité étant aussi refusée par un public qui a rapidement appris au cours de la seconde moitié du XX $\mathrm{X}^{\mathrm{e}}$ siècle à espérer «le beurre et l'argent du beurre». Et la transparence, théoriquement souhaitée par tous, ne ferait que rendre le processus plus difficile.

\section{«Dans nos sociétés, un rationne- ment éventuel est une dimension de l'équité.»}

\section{Les attentes du public et I'influence des médias}

Ici est en cause, on ne peut qu'être d'accord avec Callahan, «l'attente populaire qu'en principe les bénéfices du progrès médical doivent être accessibles à chacun indépendamment des coûts. Cette vue est celle de nombreux médecins et est encouragée par une industrie de la recherche toujours prête à claironner les progrès qu'elle apporte, laissant entendre que de nouveaux traitements décisifs et autres panacées se trouvent juste après le prochain virage. Cette attente populaire n'est pas corrélée avec la volonté de payer pour lesdits bénéfices.» Manifestement, ce n'est pas un problème américain seulement.

Il faut compter avec les effets médiatiques. Sans qu'on puisse leur interdire de le faire - et sans moyen efficace de les convaincre de renoncer à mettre l'accent sur le sensationnel -, l'expérience quotidienne montre comment les médias sont prompts à monter en épingle telle situation difficile d'une personne ou d'une famille à laquelle un traitement «héroïque» est refusé. En Suisse à fin 2010 et début 2011, la presse s'est fait largement l'écho d'un arrêt du Tribunal fédéral qui a donné raison à une caisse-maladie qui refusait de prendre en charge un médicament très cher (Myozyme) qui apportait des bénéfices de santé limités. Il a même indiqué qu'on pouvait envisager une limite de dépense de l'ordre de 100000 francs par année de vie gagnée, ce qui a logiquement fait ressurgir sur la scène nationale le thème du rationnement [6].

A l'inverse, les médias mettent moins d'empressement à vanter les mérites de mesures coupant dans des dépenses évitables et permettant la rationalisa- 
tion du système de santé (rationalisation qui, dans la pratique, peut au reste être difficile à distinguer du rationnement [7]).

\section{En guise de conclusion}

«Je n'arrive pas - dit Callahan - à imaginer qu'un rationnement explicite sera possible. Les choix difficiles seront faits sous forme de rationnement indirect ou caché, qui ne dit pas son nom. Idéalement, le seul

\section{«Le refus de nos politiciens de parler ouvertement de la nécessité du rationnement n'est pas moins pathologique.»}

rationnement (civiquement) acceptable serait fait par des comités aux compétences larges et indiscutables, et avec une contribution considérable du grand public. Mais jusqu'ici ce modèle n'a pas été pris au sérieux dans le monde politique.»

L'opinion de ce sociologue et éthicien reconnu m'a intéressé dans la mesure où, une forme de rationnement m'apparaissant inévitable, j'ai souvent espéré qu'il pourrait être prévu, cas échéant mis en œuvre, de manière explicite et démocratique, discutée sur la place publique, comme d'autres options de santé. En faisant, en Suisse, appel à la culture civique qui est la nôtre. Culture qui est réelle mais, sur le point particulier d'un rationnement transparent, je ne crois plus qu'elle soit prête à admettre la nécessité d'en débattre.

En fait, le rationnement est déjà avec nous à certains égards. Mais au niveau micro (lit du malade) ou méso (hôpital ou autre service de soins), peut-être restera-t-il ce qu'il est aujourd'hui, informel, basé sur le jugement des médecins voire d'autres soignants en contradiction avec le développement des droits des patients depuis les années 1980, qui comportent l'exigence d'une information préalable et complète. Cette situation soulève des craintes d'arbitraire et elle fait reposer des décisions lourdes de conséquences sur les équipes médicales, qui de manière compréhensible n'en veulent pas. Encore qu'il ne soit pas sûr que, au quotidien, cette informalité représente la plus mauvaise manière. Serait-ce un exemple de plus, peut-être, qu'il y a des sujets que la loi ou les réglementations ne savent pas traiter adéquatement? Où il vaudrait mieux faire appel à l'expérience et aux compétences professionnelles? Evoquant cela, je donne l'impression de vouloir réinstaller les médecins comme des «demi-dieux en blanc sur leur piédestal», au-dessus du commun, avec le risque de tromper la confiance du malade. Ce n'est certainement pas mon option mais, dans notre domaine, une longue observation me fait penser que plus de droit et de lois ne signifie pas forcément plus de raison, plus de bon sens. Depuis quelques décennies, nos régimes ont beaucoup passé en matière de santé de l'éthique au droit; il me semble indiqué dans certains cas de discuter d'un retour, du droit vers l'éthique; la relation thérapeutique soigné-soignant permet mieux que d'autres approches de prendre des décisions tenant compte de l'ensemble d'une situation personnelle.

\section{Références}

1 Callahan D. Rationing: Theory, Politics, and Passions. Hastings Center Report. 2011;41. no. 2, 23-7.

2 Kocher G. 10 Jahre Rationierungsdebatten in der Schweiz - eine Übersicht und eine vorläufige Bilanz. In: Zimmermann M, Halter H. Rationierung und Gerechtigkeit im Gesundheitswesen. Basel: EMH Schweizerischer Ärzteverlag; 2007.

3 Paccaud F, Santos-Eggimann B. Le rationnement au sein du système de santé suisse: analyse et recommandations. Bâle: Académie suisse des sciences médicales; 2007.

4 Edgar W. Rationing in health care - A New Zealand perspective on a international dilemma. Bull Méd Suisses. 2000; 81(4):190-4

5 Martin J. L'erreur serait de refuser de parler ouvertement, sereinement, du rationnement - Que nous apprend l'expérience néo-zélandaise? Bull Méd Suisses. 2000;81(4):177-8.

6 Voir entre autres articles de J.-C. Péclet, Le Temps (Genève), 29 décembre 2010, et de I. Städler, Tages-Anzeiger (Zürich), 15 avril 2011 (arrêt 9C_334/2010 du Tribunal fédéral concernant le médicament Myozyme)

7 Martin J. Le rationnement - Un point de vue d'éthique sociale et de santé publique. VSAO Journal ASMAC; 2004;23(9):39-41. 